Methodenfragen in Fachkreisen. Nicht einmal das ZAW-Rahmenschema wird erwähnt. Man ist versucht, im Vergleich nach den methodischen Standards und Kontrollen in der akademischen Publikumsforschung zu fragen.

Meyen hat sich erkennbar bemüht, allen speziellen Anforderungen an ein Lehrbuch Rechnung zu tragen - mit Erfolg. Einer der besonderen Vorzüge des Textes ist seine sprachliche Gestalt. Meyen schreibt sehr verständlich, flüssig und lebendig, regt immer wieder zum Mitdenken an (ein häufig eingesetztes Stilmittel ist die Frageform). Auch das Layout ist ansprechend: Auf vielen Seiten sorgen Aufzählungen, Tabellen oder Abbildungen für Übersicht und Abwechslung. Zentrale Punkte werden oft in Texttabellen nochmals zusammenfassend dargestellt. Allerdings entspricht die Reihenfolge der Punkte in diesen Übersichten manchmal nicht der Reihenfolge im Text. Einige der Übersichten sind nicht mit den Ausführungen im Text verknüpft; andere sind in den Text eingefügt, lange bevor dort auf sie verwiesen wird.

Die Gliederung ist sinnvollerweise einfach gehalten; innerhalb der Kapitel - die bei der Fülle von behandelten Themen manchmal ziemlich heterogen ausfallen - werden zusätzlich Zwischenüberschriften eingesetzt. An einigen Stellen aber wirkt die Strukturierung unpräzise bzw. kommt es zu fragwürdigen $\mathrm{Zu}-$ ordnungen. So werden in Kapitel 1.1 unter dem Uses-and-Gratifications-Ansatz auch zahlreiche andere eigenständige Theoriebereiche behandelt (z. B. Erregungstheorien); in Kapitel 3.1 ist Unterhaltung eine sehr breite Kategorie, die hier offenbar auch z.B. die ZeitfüllerFunktion einschließen soll (die in dem Kapitel übrigens von der Zeitstrukturierungsfunktion getrennt thematisiert wird).

$\mathrm{Zu}$ Beginn jedes Hauptkapitels wird ein Überblick über die Inhalte und Vermittlungsziele gegeben. Am Kapitelende finden sich Fragen und Aufgaben zur Selbstkontrolle (manche fordern auch - oft ausgehend von aktuellen Untersuchungsergebnissen - zum Weiterdenken auf und verlangen Transferleistungen) sowie kommentierte Literaturhinweise. Die jeweils zwei bis vier Angaben zur vertiefenden Lektüre sind zumeist sinnvoll ausgewählt, die Kommentierungen aussagekräftig. Hilfreich erscheinen auch die Hinweise auf einschlägige Periodika (einschließlich Online-Quellen), etwa für Mediennutzungsdaten. Vor allem bei den längeren Kapiteln wären allerdings mehr
Literaturangaben (nicht unbedingt alle kommentiert, ggf. sogar nur in Kurzform) wünschenswert. So hätten im vierten Kapitel Literaturhinweise zu den einzelnen Medien gegeben werden können. Auch im Text fehlen an manchen Stellen Literaturangaben zu spezifischen Ansätzen oder Methoden; eine gezielte Vertiefung anhand der Spezialliteratur ist daher nicht immer möglich. Gelegentlich bleiben auch empirische Aussagen (wie z. B. zum jahreszeitlichen Verlauf der Fernsehnutzung; S. 131) unbelegt. Im Literaturverzeichnis schließlich fehlen am Ende einige Titel.

Verschiedene Register (Sachen, Personen, Abkürzungen, Abbildungen) machen das Buch benutzerfreundlich, wenngleich sie im Detail nicht durchweg überzeugen: Im Sachregister fehlen einige zentrale Begriffe der Mediaforschung, andererseits sind viele eher überflüssige Dinge aufgeführt (etwa einzelne Zeitungstitel, die im Text nicht weiter wichtig sind). Das Abkürzungsverzeichnis scheint wie das Personenregister auf Vollständigkeit angelegt und enthält auch Einträge, die kaum jemand wird nachschlagen müssen.

Insgesamt gesehen hat Meyen mit dieser Überblicksdarstellung ein zwar durchaus verbesserungsfähiges, aber bereits jetzt in vielen Punkten vorbildliches Lehrbuch zur Mediennutzungsforschung vorgelegt. Auch fortgeschrittene Leser dürften darin noch die eine oder andere neue Erkenntnis finden.

Wolfram Peiser

\section{Annette Rinck}

\section{Interdependenzen zwischen PR und Journa- lismus}

Eine empirische Untersuchung der PR-Wirkungen am Beispiel einer dialogorientierten PR-Strategie von BMW

Wiesbaden: Westdeutscher Verlag, 2001. $325 \mathrm{~S}$.

\section{ISBN 3-531-13561-9}

Die kommunikationswissenschaftliche Forschung, die die Beziehung zwischen PR und Journalismus zum Thema macht, beschreibt und analysiert verschiedene Muster der Beeinflussung und der wechselseitigen Abhängigkeit. Die Frage nach dem „Erfolg“ dieser Beziehung für die Öffentlichkeitsarbeit stellt sich aus dieser Perspektive nicht. 
Genau an dieser Frage aber setzt Annette Rinck an. Mit der Leipziger Dissertation liegt eine Studie vor, die das Verhältnis zwischen PR und Journalismus unter dem Gesichtspunkt der PR-Erfolgskontrolle untersucht. Das Anliegen der Arbeit lässt sich in der praxisrelevanten Frage zusammenfassen: Werden die von der PR unter Berücksichtigung journalistischer Produktionsformen bereitgestellten Informationen von Journalisten im Sinne der Kommunikationsziele des PR-Treibenden genutzt? Rinck, die in der Kommunikationsabteilung bei BMW tätig ist, macht sich ihre Berufspraxis zunutze und untersucht diese Forschungsfrage am Fallbeispiel BMW. Genauer: Sie untersucht den Erfolg der Kommunikationsaktivitäten der BMW AG am Beispiel des „Verkehrskonzepts für Regensburg“. Um sich als „Unternehmen Mobilität“ in der Öffentlichkeit zu präsentieren, hatte BMW im Jahr 1994 eine Reihe von Kommunikationsmaßnahmen initiiert, darunter eine Anzeigenkampagne, Pressekonferenzen und eine Ausstellung. Im Endergebnis bestätigt Rinck mit einigen Einschränkungen den Erfolg dieser Kommunikationsaktivitäten. Da es sich um eine Einzelfallstudie handelt, ist der Aussagebereich dieses Ergebnisses eingeschränkt. Der Ertrag der Arbeit ist dann auch weniger in den Befunden, als vielmehr im Bereich der Methode zu suchen: Rinck hat ein elaboriertes Verfahren der Medienresonanzanalyse entwickelt, das sich auch in anderen Anwendungskontexten bewähren könnte.

Theoretisch orientiert sich die Autorin am Intereffikationsmodell. Dieses konzipiert das Verhältnis zwischen PR und Journalismus als ein von gegenseitigen Einfluss- und Anpassungsprozessen, so genannten Induktionen und Adaptionen, geprägtes. Damit setzt sich das Modell von der unter dem Etikett Determinationshypothese bekannt gewordenen Forschungsrichtung $a b$, die den Einfluss von PR auf Journalismus untersuchte und im Kern der PR die Fähigkeit attestierte, Themen und Timing der Berichterstattung zu kontrollieren. Im Unterschied zu dieser Herangehensweise, die das Verhältnis zwischen PR und Journalismus als ein von Macht geprägtes Verhältnis erfasst, fokussiert das Intereffikationsmodell auf die Wechselseitigkeit der Beziehungen und die Frage danach, wie sich PR und Journalismus gegenseitig ermöglichen. Gekoppelt an diesen Theorieansatz ist ein PR-Verständnis, das die
Herstellung von Vertrauen zum wesentlichen Ziel der PR erklärt.

Entsprechend dieser theoretischen Verortung, die kombiniert wird mit dem Anwendungsbezug der Fragestellung, fügt Rinck Ansätze zur dialogorientierten Unternehmenskommunikation, das Intereffikationsmodell und das Strategiemodell der PR, zu einem theoretischen Rahmen zusammen. Diese Zusammenführung stößt dort an ihre Grenzen, wo „Dialog“ zum Synonym für Adaptionsleistungen der PR hinsichtlich des Journalismus wird. Unbestritten sind Kenntnisse über journalistische Arbeitsweisen auf Seiten der PR ein entscheidender Faktor, um die Wahrscheinlichkeit zu erhöhen, dass Pressemitteilungen übernommen werden. Aber es scheint doch einigermaßen verwegen, die Vorwegnahme der Medienlogik auf Seiten der PR, die damit ihre Durchsetzungskraft erhöhen will, als „Dialog“ zu bezeichnen. Nur innerhalb des betriebswirtschaftlichen Ansatzes des gesellschaftsorientierten Marketings ist Rincks Schlussfolgerung, integrierte Unternehmenskommunikation sei eine Voraussetzung dialogischer PR, plausibel. Aus publizistik- und kommunikationswissenschaftlicher Sicht ist dieser Schluss durchaus anfechtbar, denn allzu häufig verwischen gerade in Modellen integrierter Kommunikation die Grenzen zwischen PR, Marketing und Werbung. Störend ist ferner, das sei am Rande noch vermerkt, die undifferenzierte Zitation von Praktikerliteratur und wissenschaftlicher Literatur im Theorieteil der Arbeit. Die theoretische Argumentationsführung hätte gewonnen, wäre die Unterschiedlichkeit der Erkenntnisinteressen von Wissenschaft und Praxis berücksichtigt worden.

Kernstück des aus drei Einzeluntersuchungen bestehenden empirischen Teils der Dissertation ist die von der Autorin als Input-Output-Analyse bezeichnete Inhaltsanalyse. Die Bezeichnung Input-Output-Analyse ist jedoch irreführend; denn nicht der PR-Input, sondern nur die Medienberichterstattung, die auf den PR-Input zurückzuführen ist, wird einer detaillierten empirischen Inhaltsanalyse unterzogen. Wir haben es hier also mit einer klassischen Medienresonanzanalyse zu tun. Mit dieser Analyse löst Rinck ein, was von vielen PRPraktikern gefordert, aber selten umgesetzt wird: Als Instrument zur Messung des PR-Erfolges muss eine Medienresonanzanalyse quantitativ wie qualitativ stichhaltige und in Zahlen 
bzw. in Prozenten ausdrückbare Erfolgsmaßstäbe liefern. Mit der Entwicklung eines so genannten Modifikations-/Transferindex erfüllt Rinck diese Forderung. Gemessen wurde in den insgesamt 39 untersuchten Artikeln nicht nur das Vorkommen von PR-induzierten Themen, sondern auch, auf der Ebene so genannter Informationseinheiten, die qualitative Verwendung von Kernaussagen, die auf den PR-Zielsetzungen basieren. Der Modifikations-/ Transferindex misst nun die Verteilung der Informationseinheiten der BMW-Kernaussagen im Verhältnis zu sonstigen PR-induzierten Aussagen und im Verhältnis zur journalistischen Eigenrecherche. So errechnet Rinck die prozentualen Anteile der Informationseinheiten, die von BMW als Kernaussagen definiert wurden, im Verhältnis zu den Quellen (Pressemitteilung, sonstige BMW-Informationen, journalistische Eigenrecherche). Interessant ist dabei weniger das genaue Zahlenverhältnis, das sich ja nur auf den Einzelfall bezieht und daher keine allgemeinen Aussagen über PR-Induktionen zulässt. Aber die durchgeführte $\mathrm{Me}-$ dienresonanzanalyse zeigt eine Möglichkeit auf, wie Kommunikationserfolg operationalisiert werden kann, um den Anforderungen nach messbaren Ergebnissen zu genügen.

Als zweite Teiluntersuchung hat Rinck eine Journalistenbefragung durchgeführt. Die schriftliche Befragung der Journalisten stützt bisherige Befunde: Die Skepsis gegenüber der PR und die Behauptung, Eigenrecherche sei die häufigste Informationsquelle, bestätigt das journalistische Selbstbild, das die Journalismusforschung immer wieder aufzeigt. Was Rinck als „Inhomogenität" ihrer Forschungsergebnisse wertet, ist ein neuerlicher Beleg für die Differenz zwischen journalistischem Anspruch und journalistischem Tun.

Drittens analysiert Rinck den Response auf eine BMW-Anzeigenkampagne zum Thema Mobilität. Hatten Medienresonanzanalyse und Journalistenbefragung das Problemfeld Journalismus-PR zum Gegenstand, so verlässt Rinck mit der Untersuchung der Werbewirkung ihren eigentlichen Forschungsbereich. An dieser Stelle spätestens wird deutlich, dass das vornehmliche Erkenntnisinteresse der Arbeit entgegen dem Buchtitel, der eine Untersuchung $\mathrm{zu}$ „Interdependenzen zwischen PR und Journalismus" verspricht - darin besteht, eine im Rahmen der integrierten Unternehmenskommunikation durchgeführte Imagekampagne mit den Mitteln der empirischen Sozialforschung auf ihren Erfolg hin zu bewerten. Vor allem das zu diesem Zweck entwickelte anspruchsvolle Verfahren der Medienresonanzanalyse bietet Anregungen für die weitere Forschung und Praxis. Die dabei gewonnenen Ergebnisse hinsichtlich der Verwertung von PRMaterial durch Journalisten beantworten die durch das Intereffikationsmodell aufgeworfene Frage nach einer Wechselseitigkeit der beiden Systeme PR und Journalismus allerdings nicht.

Juliana Raupp

\section{Bernd Holznagel/Andreas Grünwald \\ Meinungsvielfalt im kommerziellen Fernse- hen}

Medienspezifische Konzentrationskontrolle in Deutschland, Großbritannien, Frankreich, Italien, den USA und auf der Ebene von Europarat und Europäischer Gemeinschaft

Berlin: VISTAS Verlag, 2001. - 169 S.

(Schriftenreihe der Landesmedienanstalten, Bd. 19)

ISBN 3-89158-303-6

Digitalisierung und Konvergenz haben in der Medienpolitik in den letzten Jahren viel von sich reden gemacht. Wir haben kühne Prophezeiungen über „Individualisierung“ gehört. Eine Welle von Technikeuphorie, Freiheitspathos, Gründungsfieber, Marktgläubigkeit ist über das Land hinweggegangen. Die öffentlich-,,dienende“ Rundfunkfreiheit bisheriger (Karlsruher) Provenienz hat man in Deutschland im neu erwachten Privatisierungseifer immer wieder für überholt und veraltet erklärt. Für ein künftiges, einheitlich konzipiertes Multimedia-Recht hat man den Übergang zu presseähnlichen, möglichst geringen Regulierungsgraden gefordert. Man war von den einfachen ökonomisierenden Lösungen angetan: weg vom Kultur- und hin zum allgemeinen Wirtschaftsrecht. Hierzu gehörte auch das Bestreben, die rundfunkspezifische Konzentrationskontrolle abzuschaffen und das von ihr bisher beackerte Feld nunmehr zur Gänze der Kartellaufsicht zu überlassen. Dabei wäre es also den gegenwärtig nach $\mathbb{S} 35 \mathrm{ff}$. Rundfunkstaatsvertrag (RStV) auf dem privaten Sektor zuständigen Organen der Rundfunkaufsicht an den Kragen gegangen, vor allem der - bei den Interessenten wenig belieb- 